\title{
Engineering Disulphide-Free Autonomous Antibody VH Domains to modulate intracellular pathways
}

\section{Yuri Frosi}

p53lab, A*STAR

Shimin Jiang

Insilico Medicine Taiwan Ltd

Shimin Jiang

p53lab, A*STAR

Siti Radhiah Ramlan

p53lab, A*STAR

Kelly Hew Hew

DotBio, Pte. Ltd.

Alf Henrik Engman

DotBio Pte. Ltd.

Anil Pillai

DotBio Pte. Ltd.

\section{Yue Xiang Cheng}

DotBio Pte. Ltd.

\section{Tobias Cornvik}

Cytiva, Björkgatan 30, Uppsala, 751 84, Sweden.

\section{Pär Nordlund}

Karolinska Institutet https://orcid.org/0000-0002-7794-702X

\section{Megan Goh}

A*STAR https://orcid.org/0000-0001-7260-0601

\section{Dilraj Lama}

Department of Oncology and Pathology, Karolinska Institutet

\section{Chandra S Verma}

BII, A*STAR

\section{Dawn Thean Thean}

p53lab, A*STAR

David P Lane

p53lab, A*STAR

Ignacio Asial

DotBio Pte. Ltd.

Christopher Brown ( $\sim$ cjbrown@p53lab.a-star.edu.sg ) 
p53lab, A*STAR

Article

Keywords: proteins, intracellular pathways, VH domains

Posted Date: March 16th, 2021

DOI: https://doi.org/10.21203/rs.3.rs-244469/v1

License: (1) (1) This work is licensed under a Creative Commons Attribution 4.0 International License. Read Full License 


\section{Abstract}

An attractive approach to target intracellular macromolecular interfaces is to design small high affinity proteins. In this manuscript a stable, autonomous, human derived non-immunogenic, disulphide-free $\mathrm{VH}$ domain, has been engineered for intracellular expression studies. VH domains can be designed to possess a large dynamic repertoire of binders, as opposed to other scaffolds types that are highly rigid and possess fewer sites of random variation. Picomolar inhibitors were identified using phage display against the elF4F complex, which is commonly hyper-activated in many cancers. These molecules were also shown to impair cellular proliferation and to reduce the expression of malignancy related proteins. Structural characterization elucidated that these VH domains bound elF4E at the elF4G interaction interface via a novel binding pose. Molecules able to mimic this pose and interfere with the elF4F complex are potentially important for wide-ranging tumour therapy applications.

\section{Introduction}

Many intracellular macromolecular interfaces exist in human cancers that are highly desirable targets for the development of anti-cancer therapeutics, ranging from myc:max and b-catenin:TCF to KRAS:RAF ${ }^{1}$. Some of these interactions present significant hurdles to small molecule development due to the underlying nature of their molecular surfaces. An attractive approach to circumvent these issues is to design and evolve small high affinity proteins (approx. 60 to 100 amino acids in size) to target these interfaces, termed mini-proteins ${ }^{2,3}$. However, they are of special interest as they can easily be expressed within mammalian cells and engineered into transgenic mice, where they can be induced in a temporal and tissue specific manner. In the absence of pharmacological specific reagents they offer a precise biological tool for the interrogation of fundamental biology and target validation ${ }^{4}$.

Mini-proteins have several advantages over traditional knock-down and knock out strategies to validate candidate target proteins for therapeutic development, such as they more closely model the fundamental interactions of drugs with their targets and can also be designed to interact at the desired target sites ${ }^{5}$. Additionally, mini-proteins can be used in animal models to study potential mechanisms of toxicity and establish attainable therapeutic thresholds in conjunction with disease models ${ }^{5}$. These types of studies will allow earlier identification of relevant clinical indications for pharmacological intervention enabling the streamlining of future therapeutic development. Many currently used miniproteins are limited as binding modules due to randomization of highly rigid structural motifs with limited sites of variation. ${ }^{2}$

Variable domains of the human immunoglobulin heavy chain (VH domains) are ideal candidates for use as mini-proteins. Although of slightly larger size at approximately 120 amino acids, they possess three binding loops of variable length (CDR-H1, CDR-H2 and $\mathrm{CDR}-\mathrm{H} 3)$ that are naturally randomised to generate a wide repertoire of binders for antigen recognition by the immune system. Additionally, they should have negligible issues with immunogenicity issues in human therapy. However, the development of these scaffolds as single domain binding modalities has been restricted by poor stability, due to the loss of stabilizing interactions with the light-chain in the intact antibody 6,7 . Several groups have identified 
monomerically stable $\mathrm{VH}$ domains either by serendipitous discovery or by using phage-based evolution methods with the $\mathrm{VH}$ domain of trastuzumab (4D5) as a template. ${ }^{6,8}$ Despite the progress in the generation of an autonomous, stable $\mathrm{VH}$ domain, there has been no success in generating a stable disulphide-free $\mathrm{VH}$ domain (DiF-VH), which can be used for intracellular applications.

In this manuscript we describe the use of $\mathrm{CoFi}^{10}$ and $\mathrm{Hot}^{-\mathrm{CoFi}}{ }^{11}$ screening technologies to generate a stable, autonomous, disulphide-free VH domain (DiF-VH) based on the 4D5 antibody scaffold by directed evolution. This was then used to develop a novel $\mathrm{VH}$ domain (VH-S4) with picomolar affinity against the well-known oncogene elF4E, which was suitable for intracellular expression and activity modulation studies. elF4E forms part of the elF4F complex, which performs a critical role in mediating cap-dependent protein synthesis ${ }^{12}$. The components (elF4E, elF4A and elF4G) of which are also frequently overexpressed and/or mis-regulated in many tumours and often associated with poor prognosis in patients and chemoresistance ${ }^{13-15}$. The disulphide-free $\mathrm{VH}$ domain technology presented here offers a rapid and efficient pipeline to discover new binding poses for therapeutic lead development, as well as the identification of modalities that can be used to therapeutically model and validate these sites for drug development in mammalian and animal systems.

\section{Results}

\section{Generation of a stable, disulphide-free, autonomous antibody variable domain for intracellular expression.}

The 4D5 VH domain was chosen as the parental scaffold for stabilization, as its parental IgG trastuzumab is an approved FDA drug with a favourable immunogenic and stability profile. ${ }^{6}$ The 4D5 VH domain was fused to an $\mathrm{N}$-terminal signal sequence for periplasmic expression in $E$. coli and a random mutagenesis library of the VH ORF was created. This was then used to initiate 2 rounds of selection using Hot-CoFi ${ }^{11}$ (Fig. 1A and 1B), resulting in the isolation of 53 stabilized $\mathrm{VH}$ domain variants. Among these, $\mathrm{VH}-36$ and $\mathrm{VH}-36$ were the most thermally stable, with $\mathrm{T}_{\mathrm{CAGG}} \mathrm{S}$ of $73.4^{\circ} \mathrm{C}$ and $72.9^{\circ} \mathrm{C}$, respectively, an increase of over $20^{\circ} \mathrm{C}$ in comparison to the parent WT clone (Fig. 1C). The thermostable VH-36 clone was then selected as the starting template for the evolution of a disulphide-free VH domain suitable for intracellular cell expression. Three residues in VH-36, C22 and C92, as well as the core residue A24, were randomised with all 20 amino acids to generate a library suitable for screening. The signalling peptide, stll, was also removed from the $\mathrm{VH}-36$ expression construct (termed $\mathrm{VH}-36 \mathrm{i}$ ) to ensure expression in the cytoplasm and allow screening for autonomously stable VH domains in a cellular reducing environment. This library was screened using the Hot-CoFi methodology and five unique variants of VH-36i were identified (Fig. 1A and 1C). These were then expressed and purified, and their thermal point of denaturation ( $\left.T_{m}\right)$ determined by differential scanning fluorimetry (DSF), with VH36i.1 demonstrating the highest thermal stability with a $\mathrm{T}_{\mathrm{m}}$ of $58^{\circ} \mathrm{C}$. VH36i. 1 possessed the mutations $\mathrm{C} 22 \mathrm{~S}, \mathrm{~A} 24 \mathrm{C}$ and $\mathrm{C} 92 \mathrm{~T}$ (Fig. 1A and 1C).

\section{Optimization of a disulphide-free autonomous antibody variable domain for phage display.}


The $\mathrm{VH}-36 \mathrm{i} .1$ clone and its parent templates $(\mathrm{VH}-36$ and $\mathrm{VH}-36 \mathrm{i})$ all contained the mutation $\mathrm{A} 100 \mathrm{bP}$ within the CDR-H3 loop. An undesirable feature with respect to target binding, as it restricts the conformational space open to randomized CDR-H3 loop regions and in turn decreases their probability of interacting successfully with target molecules. Therefore, the CDR-H3 region from position W95 to P100b was removed and replaced by the sequence SSSA, creating the new construct $\mathrm{VH}-37 \mathrm{i}$, which had significantly reduced thermal stability as determined by $\mathrm{DSF}\left(\mathrm{T}_{\mathrm{m}}=46^{\circ} \mathrm{C}\right)$. VH-37i, was then used to create a random mutagenesis library for $\mathrm{CoFi}$ screening ${ }^{10}$ to compensate for this loss of protein stability. From this process 4 variants were identified with $\mathrm{VH}-37 \mathrm{i} .1$ and $\mathrm{VH}-37.2$ exhibiting the highest thermal stabilities $\left(T_{m}\right)$, approximately $55.7^{\circ} \mathrm{C}$ for both (Fig. $1 \mathrm{~A}$ and $1 \mathrm{C}$ ). Both clones contained a single mutation with $\mathrm{VH}-$ $37 \mathrm{i} .1$ bearing a $\mathrm{A} 78 \mathrm{~V}$ mutation in the core of the $\mathrm{VH}$ domain, whilst $\mathrm{VH}-37.2$ contained the mutation $\mathrm{G} 93 \mathrm{~V}$ located near the CDR-H3 loop (Fig. 1D). Both mutations were combined to generate VH38i with a $T_{m}$ of approximately $57.3^{\circ} \mathrm{C}$. To remove the final cysteine in $\mathrm{VH}-38 \mathrm{i}$ at position 24 it was either mutated to the Ala residue found in the WT template, Ile, Val, Tyr, or Trp. The VH-38i variants containing either $\mathrm{C} 24 \mathrm{I}$ or $\mathrm{C} 24 \mathrm{~L}$ substitutions were the most stable with improved $\mathrm{T}_{\mathrm{m}} \mathrm{s}$ of $58.3^{\circ} \mathrm{C}$ and $58.3^{\circ} \mathrm{C}$, respectively compared to the VH-38i template molecule (Fig. 1C).

\section{VH Domain Phage Display library construction and Delineation of the $\mathrm{VH}-1 \mathrm{~A} 2$ and $\mathrm{VH}-1 \mathrm{C} 5$ interaction sites on elF4E}

A library of VH domains were then displayed on the plll protein of M13 phage, where the CDR1 and CDR2 of the $\mathrm{VH}-38 \mathrm{i}$ template were randomized conservatively, whilst CDR3 was randomized with sequences of different lengths and biased towards residues serine and tyrosine with approximately $20 \%$ frequency each. Phage display selection against purified human full-length elF4E led to the identification of $\mathrm{VH}$ Domains VH-1A2 and VH-1C5 as the highest affinity binders with $\mathrm{K}_{d} \mathrm{~S}$ of $115.2 \pm 4.4 \mathrm{nM}$ and $154.3 \pm$ 70.3nM, respectively (Fig. 2A, Table 1). The phage selected CDR3 loop sequences of the VH-1A2 and VH$1 \mathrm{C} 5$ domains shared little similarity to the interacting motifs of known elF4E binding proteins such as elF4G1 and the 4E-BP family (YXXXXLФ, termed the canonical motif) (Fig. 2B). Competitive based fluorescence anisotropy experiments were performed that mapped the binding of $\mathrm{VH}-1 \mathrm{~A} 2$ and $\mathrm{VH}-1 \mathrm{C} 5$ to the elF4G interaction site (Fig. 2B). 
Table 1

Binding and kinetics parameters of various VH domains against elF4E. $\mathrm{K}_{\mathrm{d}}, \mathrm{k}_{\mathrm{on}}$ and $\mathrm{k}_{\mathrm{off}}$ values were derived from SPR experiments using either single cycle or multi cycle injection experiments against elF4E amine coupled to a CM5 sensorchip. Parameters were derived using a 1:1 site binding model. However, with regards to $4 \mathrm{E}-\mathrm{BP} 1^{4 \mathrm{ALA}}$, binding parameters were initially determined using a 1:1 site binding model, which generated a poor fit and physically irrelevant $\mathrm{K}_{\text {on }}$ and $\mathrm{K}_{\text {off }}$ values beyond the detection limit of the machine. This analysis was superseded with a 2-state analysis, which generated more meaningful kinetic parameters. The use of a 2-state model is supported by following reasons: 1) $4 \mathrm{E}-\mathrm{BP} 1^{4 \mathrm{ALA}}$ undergoes a

disorder to order transition upon binding elF4E and, 2) it interacts at elF4E through 2 binding sites.

\begin{tabular}{|c|c|c|c|c|c|}
\hline & $K_{d}(n M)$ & \multicolumn{2}{|c|}{$k_{\text {on }}\left(S^{-1} M^{-1}\right)$} & \multicolumn{2}{|l|}{$k_{\text {off }}\left(S^{-1}\right)$} \\
\hline $\mathrm{VH}-1 \mathrm{~A} 2$ & $115.2 \pm 4.4$ & \multicolumn{2}{|c|}{$3.43 \times 10^{5} \pm 3.75 \times 10^{4}$} & \multicolumn{2}{|c|}{$3.78 \times 10^{-2} \pm 3.17 \times 10^{-3}$} \\
\hline \multirow[t]{2}{*}{ VH-1C5 } & $\begin{array}{l}154.3 \pm \\
70.3\end{array}$ & & & $4.11 \times 10^{-2}$ & $6.99 \times 10^{-3}$ \\
\hline & & \multicolumn{4}{|c|}{$2.85 \times 10^{5} \pm 8.5 \times 10^{4}$} \\
\hline VH-1C5 $104 \mathrm{~A}$ (VH-M4) & $4.8 \pm 1.7$ & \multicolumn{2}{|c|}{$2.46 \times 10^{6} \pm 3.89 \times 10^{4}$} & \multicolumn{2}{|c|}{$1.18 \times 10^{-2} \pm 3.90 \times 10^{-3}$} \\
\hline VH-1C5 & $0.94 \pm 0.04$ & \multicolumn{2}{|c|}{$2.63 \times 10^{6} \pm 2.98 \times 10^{5}$} & \multicolumn{2}{|c|}{$2.48 \times 10^{-3} \pm 3.79 \times 10^{-4}$} \\
\hline VH-1C5 $5^{\text {D104A/F120I }}(\mathrm{VH}-\mathrm{S} 2)$ & $0.64 \pm 0.06$ & \multicolumn{2}{|c|}{$1.18 \times 10^{6} \pm 1.85 \times 10^{5}$} & \multicolumn{2}{|c|}{$7.64 \times 10^{-4} \pm 1.80 \times 10^{-4}$} \\
\hline $\begin{array}{l}\text { VH1-C5D104A/S108R/F120I } \\
\text { (VH-S4) }\end{array}$ & $\begin{array}{l}0.057 \pm \\
0.004\end{array}$ & \multicolumn{2}{|c|}{$5.18 \times 10^{6} \pm 8.55 \times 10^{5}$} & \multicolumn{2}{|c|}{$2.96 \times 10^{-4} \pm 6.47 \times 10^{-5}$} \\
\hline \multirow[t]{2}{*}{ 4E-BP1 4ALA (1:1) } & $0.60 \pm 0.14$ & \multicolumn{2}{|c|}{$1.05 \times 10^{10} \pm 1.48 \times 10^{9}$} & \multicolumn{2}{|l|}{$5.93 \pm 0.56$} \\
\hline & & $\mathrm{k}_{\text {on1 }}$ & $k_{\text {on2 }}$ & $k_{\text {off1 }}$ & $k_{\text {off2 }}$ \\
\hline \multirow[t]{2}{*}{ 4E-BP1 ${ }^{4 A L A}$ (2 state) } & $0.68 \pm 0.13$ & $8.35 \times 10^{6}$ & $1.70 \times 10^{-2}$ & $3.19 \times 10^{-4}$ & $1.84 \times 10^{-4}$ \\
\hline & & $\begin{array}{l} \pm 4.90 x \\
10^{6}\end{array}$ & $\begin{array}{l} \pm 1.08 x \\
10^{-2}\end{array}$ & $\begin{array}{l} \pm 7.91 x \\
10^{-5}\end{array}$ & $\begin{array}{l} \pm 7.00 x \\
10^{-5}\end{array}$ \\
\hline
\end{tabular}

\section{Structural Characterization Of Vh-1c5 Interaction Site With Eif4e}

Crystals were obtained for elF4E complexed with $\mathrm{m}^{7} \mathrm{GTP}$ and $\mathrm{VH}-1 \mathrm{C} 5$ (Figure S1), which revealed that VH$1 \mathrm{C} 5$ bound elF4E at a position that overlaps with the elF4G interaction site. The phage selected CDR3 loop (residues 111-120) of VH-1C5 forms a highly folded small domain structure that interacts directly with elF4E, whose structure contrasts sharply with the 'L'-shaped conformers formed by 4E-BP1 and elF4G derived linear peptides when in complex with elF4E (Fig. 2C and 2D) ${ }^{16}$. The random coil section (residues 101-107) of the CDR3 loop orientates the VH domain residue L105 into a position that mimics the interactions made by the conserved hydrophobic residue in the elF4E interaction (residues M60 and L630 in $4 \mathrm{E}-\mathrm{BP} 1$ and elF4G1, respectively) (Fig. 2E). The random coil section of the $\mathrm{VH}$ domain also forms 
additional contacts with a hydrophobic region located on elF4E unexploited by the canonical

peptides ${ }^{17,18}$ e.g., the side chain of $A 102$ forms a hydrophobic contact with W73, whilst the sidechain of D104 packs against Y76 and L131. Y117, located on the helical segment (residues 108-117) of the CDR3 loop, forms hydrophobic packing interactions with L135 and a sidechain to sidechain hydrogen bond with E132 on the surface of elF4E (Fig. 2E). These interactions replace the conserved electrostatic interaction mediated by R55 and R625 of the 4E-BP1 and elF4G1 peptides with E132, respectively. F220, positioned on the short helical turn motif (residues 119-121) that precedes the loop re-joining the main body of the $\mathrm{VH}^{1 \mathrm{C} 5}$ fold, forms several hydrophobic contacts with the elF4E residues L39, V69 and I138 and a backbone hydrogen bond interaction with the indole side chain of W73 on elF4E (Fig. 2F). These interactions are replicated by the conserved $Y$ and $L$ residues of the elF4E interaction motif ( $Y 54 / 624$ and $\mathrm{L} 59 / 629$ of $4 \mathrm{E}-\mathrm{BP} 1$ and elF4G1, respectively) with the backbone carbonyl of the $\mathrm{L}$ forming a hydrogen bond with W73.

\section{Alanine Scanning Mutagenesis Reveals $\mathrm{V}_{\mathrm{H}} 1 \mathrm{C5}$ Residues Distal to the Interaction Interface are Critical for Binding}

Alanine mutagenesis scanning of the CDR3 library insert (Fig. 3) was performed using yeast display. These experiments revealed two classes of mutants that adversely affected the binding of $\mathrm{VH}^{1 \mathrm{C} 5}$ to elF4E (Fig. 3A and $3 \mathrm{~B}$ ). The first class of mutations describe residues identified in the crystal structure that are in direct contact with elF4E (K102, A103, D104, L105, T116, Y117 and F120). These results showed high correlation with residues that were identified through computational molecular dynamics simulations to significantly contribute energetically to the elF4E:VH-1C5 complex interface (Fig. 3C and Table S1). Interestingly, both alanine mutagenesis scanning and binding decomposition analysis identified that D104 forms unfavourable interactions at the elF4E and VH-1C5 interface, and when replaced with alanine resulted in a VH-1C5 variant (VH-M4) that had an approximate 17-fold improvement in its affinity towards eif4E (Fig. 3D, Table 1) with a $\mathrm{K}_{d}$ of $4.8 \pm 1.7 \mathrm{nM}$. Inspection of the elF4E: VH-1C5 structure revealed that the residues in proximity to D104 are primarily hydrophobic and that the D104A mutation allows for more favourable van der Waals interactions to occur.

The second class of mutations describe the alanine scanning replacement of residues V101, P110, V113, V114 and F122 that abolished elF4E binding but were not located at the VH-1C5 interface. These residues form a distinct hydrophobic cluster that interact with several hydrophobic residues located on the $\beta$-sheet face of the immunoglobulin fold of the VH domain (V39, L47 and W49) (Fig. 3E). The sensitivity of VH$1 \mathrm{C} 5$ binding to elF4E when this cluster is mutated suggests that these residues play a critical role in ensuring the correct folding of the CDR3 loop and spatial orientation of the contact residues. It also implies that the CDR3 loop forms a highly stable fold, a conclusion further supported by MD simulations that shows that the CDR3 loop structure of the unbound form deviates negligibly from the bound form (Fig. 3F). A salt bridge also forms between residues R52 and D37 at the interface between the CDR3 loop and the $\mathrm{VH}$ domain fold, which orientates the $\mathrm{R} 52$ to form long range electrostatic interactions with the backbone carbonyls of S107 and S108 further rigidifying the CDR3 loop. 


\section{Evolution of an Ultra High Affinity Mini-Protein Inhibitor of the elF4E:4G interaction}

The improved VH-M4 binder $\left(\mathrm{K}_{\mathrm{d}}=4.8 \pm 1.7 \mathrm{nM}\right)$ was used as a template sequence to generate a randomly mutagenized library for affinity maturation to identify improved binders against elF4E using yeast display (Fig. 4A). Three rounds of kinetic selection were performed with increasing incubations times of 8 mins, 60 mins and 110 mins to increase the competitive pressure of unlabelled elF4E against the VH domain library complexed to fluorescent elF4E (Fig. 4B). The final round of kinetic selection resulted in the identification of only three elF4E binders: VH-1C5 ${ }^{\mathrm{D} 104 \mathrm{~A} / \mathrm{S} 108 \mathrm{R} / \mathrm{F} 1201}$, VH-1C5 $5^{\mathrm{D} 104 \mathrm{~A} / \mathrm{S} 24 \mathrm{G} / \mathrm{F} 1201}$ and $\mathrm{VH}-$

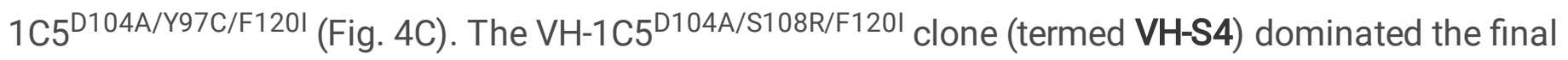
round with a frequency $>90 \%$ and was selected for scale up and SPR analysis, which revealed that it interacted with a sub-nanomolar $K_{d}$ of $0.057 \pm 0.004 \mathrm{nM}$ (Fig. 4D, Table 1). The 2 substitutions identified in the VH-S4 sequence were then individually introduced into $\mathrm{VH}-1 \mathrm{C} 5-\mathrm{M} 4$ and their binding to elF4E assessed. SPR analysis revealed that VH-1C5 D104A/S108R and VH-1C5 ${ }^{\text {D104A/F120I (termed VH-S2) }}$ possessed $\mathrm{K}_{d} s$ of $0.94 \pm 0.04 \mathrm{nM}$ and $0.64 \pm 0.06 \mathrm{nM}$, respectively (Fig. 4, Table 1). Preliminary examination and MD simulations of the $\mathrm{VH}-1 \mathrm{C} 5$ :elF4E crystal structure with the identified mutations highlighted no substantial change in the energetics profile of the interaction (Figure S2) but did indicate that the F120l mutation most likely optimised the hydrophobic contacts between both proteins and allowed a more optimal engagement to occur. However, with regards to the S108R mutation, MD simulations showed only a transient interaction with R128 of elF4E (Table S1).

The crystal structure of elF4E: VH-1C5D104A/S108R/F120I (VH-S4) complex was resolved, which enabled confirmation that the F120I mutation improved the complementarity of fit between the two proteins (Fig. 4E and S3). The elucidated complex structure also showed that the S108R mutation did not result in a direct interaction with the surface of elF4E, but rather that it engaged a structured water network that forms an h-bond to the backbone carbonyl of R128 on elF4E (Fig. 4F and S3). Additionally, S108R formed a cation- $\pi$ interaction with the sidechain of the neighbouring F112, further helping to stabilise the local fold of the CDR3 recognising elF4E. The elF4E: VH-1C5 D104A/S108R/F120I (VH-S4) crystal structure also revealed that the substitution of D104 with alanine results in a significant conformational change in Y76 upon the surface of elF4E (Fig. 4G and S3). This change is also associated with the subtle migration of several structure waters that enable the local $\mathrm{H}$-bond network around the $\mathrm{Y} 76$ residue to be maintained. The D104A mutation removes the unfavourable packing of the negatively charged D104 side chain from against Y76 and allows a more optimal packing arrangement to occur (Fig. 4G and S3).

\section{VH-S4 Disrupts elF4F Complex Formation and Cap-Dependent Translation In Vitro}

VH-S4 was expressed in HEK293 cells and shown to immune-precipitate endogenous elF4E more strongly than the $\mathrm{VH}$ domain mutants with weaker affinities (VH-1 C5, VH-M4 and VH-S2) for elF4E (Fig. 5A). A VH$1 \mathrm{C} 5$ negative control ( $\left.\mathrm{VH}-1 \mathrm{C} 5^{\mathrm{SCRM}}\right)$, where the residues in the CDR3 loop were scrambled, was also included that failed to pull-down elF4E demonstrating the specificity of the VH domains for elF4E (Fig. 5A). The ability of VH-S4 to interact with elF4E was also compared to a constitutively active 4E-BP1 
construct (termed 4E-BP1 ${ }^{4 A I A}$ ), where the phosphorylation sites (Thr37, Thr46, Ser65, Thr70) ${ }^{19}$ responsible for modulating its binding with elF4E were mutated to alanine. The $\mathrm{K}_{d}$ of $4 \mathrm{E}-\mathrm{BP} 1^{4 A L A}$ against elF4E was determined to be $0.68 \pm 0.13 \mathrm{nM}$ approximately 10 -fold weaker than the VH-S4 interaction with elF4E, with a substantially weaker off-rate (Table 1 and Figure S4). Both constructs exhibited comparable levels of activity in immuno-precipitating elF4E, whilst the 4E-BP1 $4 \mathrm{ALA}$ negative control (termed 4E$B P 1^{Y L M}$ ) had negligible effects. VH-S4 was then shown to more efficiently disrupt the elF4G-elF4E interaction in cells than the weaker affinity VH domain variants (1C5, S2 and M4) using the NanoBit elF4E:elF4G ${ }^{606-646}$ cell-based assay ${ }^{20}$ (Fig. 5B). 4E-BP1 ${ }^{4 A L A}$ was also assessed in the NanoBIT assay and again was similar in activity to $\mathrm{VH}-\mathrm{S} 4$. All the $\mathrm{VH}$-domains except the control were then shown to inhibit cap-dependent translation in a bicistronic reporter assay, with the magnitude of cap-independent inhibition again correlating to the affinity of the elF4E interacting VH domains (Fig. 5C). It is well established that disruption of the elF4F complex prevents elF4G mediated phosphorylation of elF4E by Mnk1. ${ }^{21}$ Therefore, in parallel, lysates were co-prepared from the cells used in the NanoBIT assay and the levels of phosphorylated elF4E detected (Fig. 5C). elF4E phosphorylation as expected closely followed the levels of elF4F complex disruption measured in the NanoBit elF4E:elF4G ${ }^{606-646}$ system. 4E-BP1 ${ }^{4 A L A}$ was also tested in both the bicistronic and elF4E phosphorylation assays, where it specifically decreased elF4F complex formation and phosphorylation levels in a similar manner to the VH-S4 domain (Fig. 5C).

The effects of elF4F complex disruption directly upon cap-dependent protein translation were monitored by measuring Cyclin D1 protein levels ${ }^{22,23}$. Increasing amounts of VH-M4 and VH-S4 expression plasmid were transfected into mammalian cells with a concomitant inhibitory effect on elF4E phosphorylation and cyclin D1 protein levels (Fig. 5D), with the more tightly binding $\mathrm{VH}-\mathrm{S} 4$ domain $\left(\mathrm{K}_{d}=60 \mathrm{pM}\right)$ having a more profound effect. This effect of elF4F disruption upon cyclin-D1 translation and elF4E phosphorylation was also reflected by transfection experiments with $4 \mathrm{E}-\mathrm{BP} 1^{4 \mathrm{ALA}}$ (Fig. 5E and 5F). $\mathrm{m}^{7} \mathrm{GTP}$ mediated pull down experiments, using HEK293 transfected cells, verified that all the VH domains (S4, M4, S2 and 1C5) and 4E-BP1 ${ }^{4 A L A}$, except for the controls, were able to competitively displace elF4G from elF4E and decrease the amount of endogenous elF4F complex detected (Fig. 5E).

\section{Vh-s4 Modulates Eif4f Mediated Signalling Pathways Specifically}

elF4E inhibition has been shown to reduce the expression of malignancy-related proteins (e.g., cyclin $\mathrm{D} 1^{24}, \mathrm{Mcl}^{-1}{ }^{25}$ and BCL-x| ${ }^{26-28}$ ). Additionally, the most reported biological consequences of elF4E inhibition are significant reductions in cellular proliferation and induction of apoptosis. To examine the cellular effects of $\mathrm{VH}-\mathrm{S} 4$ and $4 \mathrm{E}-\mathrm{BP} 1{ }^{4 \mathrm{ALA}}$ mediated inhibition of elF4E, stably transfected inducible expression systems were constructed for both proteins in A375 melanoma and MBA-MD-321 breast carcinoma cells. VH-S4 and 4EBP1 ${ }^{4 A l a}$ were both induced with doxycycline for 24 hours in both cell lines and were shown to dramatically decrease elF4E and elF4F complex formation (Figure S5A) in comparison to mock control cells. Both proteins when induced for over 7 days also caused significant decreases in the cellular proliferation and viability (Figs. 6A, 6B, S5C and S5D). The most dramatic effects were seen in A375 cells, where both 4E-BP1 ${ }^{4 A L A}$ and $\mathrm{VH}-\mathrm{S} 4$ decreased cellular proliferation and viability to 
the same extent (Fig. 6A and 6B). In contrast, VH-S4 was less efficacious than 4E-BP1 ${ }^{4 A L A}$ in MD-MBA231 cells, despite disrupting elF4F complex formation to the same extent (Figure S5A).

We next assessed the effects of both proteins on the expression of several proteins (Cyclin-D1, Mcl-1 and $\mathrm{Bcl}-\mathrm{xl}$ ) whose translation is regulated by the elF4F complex in A375 cells (Fig. 6C). Both VH-S4 and 4E$\mathrm{BP} 1{ }^{4 A L A}$ when induced for 24 hours resulted in significant decreases in the protein expression levels of cyclin D ${ }^{24}$ and of MCL-1 ${ }^{25}$ but had little effect on Bcl-xl ${ }^{26-28}$ levels. Similar effects on Mcl-1 and Cyclin D1 levels were observed in the MDA-MB-231 cell line (Figure S5B). Interestingly, A375 cells demonstrated no evidence of apoptosis as measured by PARP cleavage with the induction of either miniprotein, post 24 and 72 hours (Fig. $6 \mathrm{C}$ ). This result suggests that the down-regulation of Mcl- 1 is insufficient to induce apoptosis in A375 cells and that the decrease in cellular proliferation measured with both VH-S4 and 4E$\mathrm{BP} 1^{4 \mathrm{ALA}}$ is principally driven by the reduction in Cyclin-D1 protein levels (Fig. $6 \mathrm{C}$ ). Additionally, both VH-S4 and $4 \mathrm{E}-\mathrm{BP}{ }^{4 \mathrm{ALA}}$ reduced the total protein expression of $4 \mathrm{E}-\mathrm{BP} 2$ with negligible effects on $4 \mathrm{E}-\mathrm{BP} 1$ levels. Protein expression levels was reassessed post 72 hours doxycycline induction, which revealed that Mcl-1 was no longer significantly repressed but showed that 4E-BP1 levels were reduced with a concomitant decrease in its phosphorylated forms (Figure S6A). This lack of sustained decrease in Mcl-1 levels offers further explanation of the lack of cellular apoptosis with elF4F complex disruption. In parallel experiments, both $\mathrm{VH}$-domains and $4 \mathrm{E}-\mathrm{BP} 1^{4 \mathrm{ALA}}$ inhibited global protein synthesis by approximately $50 \%$ as determined using puromycin pulse chase experiments (Figure S6B). Results that correlated with the significant effects of elF4F complex disruption upon cellular proliferation (Fig. 6A). We also verified the role of elF4F in mediating STAT1 levels in IFN- $y$ treated A375 cells (Fig. 6D) ${ }^{29}$, where selective induction of $\mathrm{VH}-\mathrm{S} 4$ or $4 \mathrm{E}-\mathrm{BP} 1^{4 \mathrm{ALA}}$ in IFN- $\gamma$ treated cells reduced STAT1 protein levels.

The specificity of the VH-S4 domain was assessed by examining its effects on the pathways (AKT/mTORC and RAS/ERK) that regulate the elF4F complex. Many of the chemical reagents used to study the biological function of the elF4F complex modulate these pathways, and as a result elicit elF4F independent effects e.g. PP2 $22^{30}$, an ATP competitive inhibitor of mTORC1/2 that leads to dephosphorylation of both its downstream targets, 4E-BP1 and S6 kinase. A375 cells were treated with either PP242 or staurosporine (a non-selective kinase inhibitor) ${ }^{31}$ and their effects on elF4E, AKT, ERK and rS6 phosphorylation compared to disruption of the elF4F complex by both $4 \mathrm{E}-\mathrm{BP} 1^{4 \mathrm{ALA}}$ and VH-S4 (Fig. 6C). Both miniproteins, caused dephosphorylation of elF4E in a manner similar to PP242 through inhibition of elF4G mediated MNK1 phosphorylation. ${ }^{20}$ As expected, PP242 reduced 4E-BP1 phosphorylation to mediate its known effects on elF4F complex disruption ${ }^{30}$. However, PP242 unlike the miniproteins also reduced phosphorylation of rS6 and AKT phosphorylation though its effects on mTORC12 (Fig. 6C) ) $^{30,32}$. A375 cells treated with $0.2 \mu \mathrm{M}$ of the broad kinase inhibitor staurosporine resulted only in the dephosphorylation of S6 kinase. Neither the miniproteins nor the small molecules tested affected ERK phosphorylation.

The effects of small molecules upon the elF4F complex were extended to a wider set of compounds (including the elF4E inhibitor, 4EGI-1 and the Mnk Kinase inhibitor, CPG-57380) and assessed at 72 hours 
(Fig. 6E). The effects of PP242 and staurosporine on protein expression levels of Mcl-1 at 24 hours (Fig. 6C) were alleviated by 72 hours (Fig. 6E), whilst maintained with respect to cyclin-D1, 4E-BP1 and 4EBP2. CPG-57380 as expected resulted in dephosphorylation of elF4E at 72 hours (Fig. 6E) with negligible effects on cyclin-D1 or 4E-BP1/2 protein levels. Additionally, PP242 and staurosporine induced apoptosis at 72 hours as indicated by PARP cleavage (Fig. 6E and Figure S6C)) in contrast to the miniproteins demonstrating that specific inhibition of the elF4E: $4 \mathrm{G}$ interface does not lead to apoptosis and that this is the result of other modes of action by either small molecule. Interestingly $4 \mathrm{EGI}-1$, a molecule that allosterically disrupts binding of elF4G to elF4G, also elicits PARP cleavage and promotes elF4E phosphorylation in contrast to VH-S4 and 4E-BP1 ${ }^{4 A L A}$.

\section{Discussion}

The rapid generation of high affinity mini-proteins against protein-protein interactions intractable to small molecule development represents a valuable methodology for target validation and therapeutic modelling. We have developed a repertoire of $\mathrm{VH}$ domain-based mini-proteins that sample a wide range of $\mathrm{K}_{\mathrm{d}} \mathrm{s}$ (picomolar to micromolar), which all disrupt the elF4F complex though a novel binding pose and that are all amenable to cellular expression. A unique feature of the evolved elF4E interacting VH domains is that the randomised CDR3 loop forms a well-defined domain type structure. In contrast to PPIs such as Mdm2:p53 $3^{33}$ and $\mathrm{Bcl}-2: \mathrm{Bax}^{34}$, the elF4E: $4 \mathrm{G}^{16}$ interaction interface is relatively planar with no distinguishing clefts and is much more structurally similar to PPIs such as the interface found in the Notch transcription complex between ICN:CSL and MAML and $\beta$-catenin:TCF. ${ }^{35}$ The elF4E interacting VH domains have in effect evolved a module that is optimized for recognizing planar PPI surfaces, and with randomization of the appropriate residues that are non-critical to the stabilizing core residues, should serve as an ideal template for building libraries to explore these molecular surfaces further. Additionally, no clinically approved drugs exist that target the elF4E:4G interface directly, despite the description of several antagonistic small molecules in the literature that albeit interact weakly with elF4E and have little cellular efficacy. ${ }^{12}$ The VH-S4 and VH-1C5 structures offer two possible templates for future lead molecule development: 1 ) the CDR3 loop could be used as a template to drive forward a macrocyclic discovery strategy and 2) the critical residues involved in binding elF4E could be used as a basis for small molecule design.

The applicability of VH domains for cellular pathway modulation and therapeutic modelling were demonstrated by comparing their mode of action to several small molecules and a phosphorylation deficient mutant of $4 \mathrm{E}-\mathrm{BP} 1$ (4E-BP1 ${ }^{4 \mathrm{ALA}}$ ) known to modulate the elF4F complex. Both 4E-BP1 ${ }^{4 \mathrm{ALA}}$ and VHS4 exhibited similar potencies to each other in terms of their bioactivity, which is surprising as VH-S4 interacts more strongly with elF4E (Table 1). Both proteins also retarded cellular proliferation to a similar extent in A375 cells and exhibited a similar profile in down-regulation expression of Mcl-1, cyclin D1 and $4 \mathrm{E}-\mathrm{BP} 1 / 2$ protein levels at 24 and 72 hours. Interestingly, 4E-BP1 ${ }^{4 \mathrm{ALA}}$ was more effective in reducing cellular proliferation of MB-MDA-231 cells despite similar efficacies to VH-S4 in terms of elF4F complex disruption (Figure S5). These results suggest that other mechanisms apart from displacement of elF4G 
are putatively playing a role in the effects of $4 \mathrm{E}-\mathrm{BP} 1^{4 \mathrm{ALA}}$ mediated inhibition. Such effects could be post translational modifications apart from phosphorylation (e.g., ubiquitination of elF4E:4E-BP1 4ALA leading to increased degradation) or additional inhibition by endogenous 4E-BP1 through unappreciated feedback loops. This demonstrates that $\mathrm{VH}$ domains that are inert to cellular regulation pathways can be used to decipher their effects on naturally occurring repressors e.g., 4E-BP1, provided they interact at the same binding site.

Furthermore, the $\mathrm{VH}-\mathrm{S} 4$ domain was compared to several well-known compounds that have been used to study the biological role of the elF4F complex. For example, inhibitors of mTORC1, the multi-subunit protein responsible for phosphorylating 4E-BP1 and controlling its ability to inhibit elF4E, is also responsible for regulating the activity of other target proteins and their downstream pathways, such as the rS6 kinase and the elongation step of protein synthesis. ${ }^{36,37}$ Here we could demonstrate that VH-S4 exquisitely disrupts elF4F complex disruption in the absence of effects on rS6 kinase phosphorylation (including 4EBP1 also) and is thus a more appropriate tool for understanding the biological impact of elF4E. Additionally, we also compared it to the small molecule inhibitor of the elF4E:4G interaction, 4EGI$1^{38}$, where in stark contrast to the VH-S4 domain the induction of apoptosis was seen. A result shared with the in mTORC1 inhibitor that was used (PP242). These results further demonstrate how highly specific mini-proteins such as the VH-S4 can be used to refine our understanding of the impact of small molecule inhibition on cellular phenotypes, and to delineate whether desirable outcomes such as apoptosis are the result of direct on target engagement or indirect off-target effects. Tools that could be invaluable for therapeutic design and research.

In summary, we have generated a unique disulphide-free VH domain scaffold optimized for intracellular folding and function and demonstrated how to generate picomolar-affinity VH domains to target and inhibit therapeutically attractive intracellular PPI interfaces through a unique binding mode. This unique binding mode can be used to inspire the new design of novel modalities and small molecules. VH domain display technology therefore represents a powerful valuable tool to discover new modes of inhibition and mini-proteins for exploratory biology and therapeutic modelling against macromolecule interfaces. We envision that in the future combined with new and novel alternate delivery methods e.g., RNA, toxin, and cell-penetrating peptide mediated delivery methods, that mini-proteins could also be used to target intracellular targets of interest and constitute potential therapeutics.

\section{Declarations}

\section{Acknowledgments:}

PN gratefully acknowledge funding from a start-up grant from Nanyang Technological University and grants from the Swedish Research Council.

\section{References}


1. Petta, I., Lievens, S., Libert, C., Tavernier, J. \& De Bosscher, K. Modulation of Protein-Protein Interactions for the Development of Novel Therapeutics. Mol. Ther. J. Am. Soc. Gene Ther. 24, 707718 (2016).

2. Binz, H. K., Amstutz, P. \& Plückthun, A. Engineering novel binding proteins from nonimmunoglobulin domains. Nat. Biotechnol. 23, 1257-1268 (2005).

3. Crook, Z. R., Nairn, N. W. \& Olson, J. M. Miniproteins as a Powerful Modality in Drug Development. Trends Biochem. Sci. 45, 332-346 (2020).

4. Blagg, J. \& Workman, P. Choose and Use Your Chemical Probe Wisely to Explore Cancer Biology. Cancer Cel/ 32, 9-25 (2017).

5. Soucek, L. et al. Modelling Myc inhibition as a cancer therapy. Nature 455, 679-683 (2008).

6. Barthelemy, P. A. et al. Comprehensive analysis of the factors contributing to the stability and solubility of autonomous human VH domains. J. Biol. Chem. 283, 3639-3654 (2008).

7. Holliger, P. \& Hudson, P. J. Engineered antibody fragments and the rise of single domains. Nat. Biotechnol. 23, 1126-1136 (2005).

8. Chen, W., Zhu, Z., Feng, Y., Xiao, X. \& Dimitrov, D. S. Construction of a large phage-displayed human antibody domain library with a scaffold based on a newly identified highly soluble, stable heavy chain variable domain. J. Mol. Biol. 382, 779-789 (2008).

9. Ma, X., Barthelemy, P. A., Rouge, L., Wiesmann, C. \& Sidhu, S. S. Design of synthetic autonomous VH domain libraries and structural analysis of a VH domain bound to vascular endothelial growth factor. J. Mol. Biol. 425, 2247-2259 (2013).

10. Cornvik, T. et al. Colony filtration blot: a new screening method for soluble protein expression in Escherichia coli. Nat. Methods 2, 507-509 (2005).

11. Asial, I. et al. Engineering protein thermostability using a generic activity-independent biophysical screen inside the cell. Nat. Commun. 4, 2901 (2013).

12. Bhat, M. et al. Targeting the translation machinery in cancer. Nat. Rev. Drug Discov. 14, 261-278 (2015).

13. Boussemart, L. et al. elF4F is a nexus of resistance to anti-BRAF and anti-MEK cancer therapies. Nature 513, 105-109 (2014).

14. Malka-Mahieu, H., Newman, M., Désaubry, L., Robert, C. \& Vagner, S. Molecular Pathways: The elF4F Translation Initiation Complex-New Opportunities for Cancer Treatment. Clin. Cancer Res. Off. J. Am. Assoc. Cancer Res. 23, 21-25 (2017).

15. Zindy, P. et al. Formation of the elF4F translation-initiation complex determines sensitivity to anticancer drugs targeting the EGFR and HER2 receptors. Cancer Res. 71, 4068-4073 (2011).

16. Marcotrigiano, J., Gingras, A. C., Sonenberg, N. \& Burley, S. K. Cap-dependent translation initiation in eukaryotes is regulated by a molecular mimic of elF4G. Mol. Cel/ 3, 707-716 (1999).

17. Grüner, S. et al. The Structures of elF4E-eIF4G Complexes Reveal an Extended Interface to Regulate Translation Initiation. Mol. Cell 64, 467-479 (2016). 
18. Peter, D. et al. Molecular architecture of 4E-BP translational inhibitors bound to elF4E. Mol. Cel/ 57, 1074-1087 (2015).

19. Gingras, A. C. et al. Hierarchical phosphorylation of the translation inhibitor 4E-BP1. Genes Dev. 15, 2852-2864 (2001).

20. Frosi, Y., Usher, R., Lian, D. T. G., Lane, D. P. \& Brown, C. J. Monitoring flux in signalling pathways through measurements of 4EBP1-mediated elF4F complex assembly. BMC Biol. 17, 40 (2019).

21. Shveygert, M., Kaiser, C., Bradrick, S. S. \& Gromeier, M. Regulation of Eukaryotic Initiation Factor 4E (elF4E) Phosphorylation by Mitogen-Activated Protein Kinase Occurs through Modulation of Mnk1elF4G Interaction. Mol. Cell. Biol. 30, 5160-5167 (2010).

22. Averous, J., Fonseca, B. D. \& Proud, C. G. Regulation of cyclin D1 expression by mTORC1 signaling requires eukaryotic initiation factor $4 \mathrm{E}$-binding protein 1. Oncogene 27, 1106-1113 (2008).

23. Culjkovic, B., Topisirovic, I. \& Borden, K. L. Controlling gene expression through RNA regulons: the role of the eukaryotic translation initiation factor elF4E. Cell Cycle 6, 65-69 (2007).

24. Mamane, Y. et al. elF4E--from translation to transformation. Oncogene 23, 3172-3179 (2004).

25. Wendel, H.-G. et al. Dissecting elF4E action in tumorigenesis. Genes Dev. 21, 3232-3237 (2007).

26. Polunovsky, V. A. et al. Translational control of the antiapoptotic function of Ras. J. Biol. Chem. 275, 24776-24780 (2000).

27. Li, S. et al. Translational control of cell fate: availability of phosphorylation sites on translational repressor 4E-BP1 governs its proapoptotic potency. Mol. Cell. Biol. 22, 2853-2861 (2002).

28. Li, S. et al. Translation initiation factor $4 \mathrm{E}$ blocks endoplasmic reticulum-mediated apoptosis. J. Biol. Chem. 279, 21312-21317 (2004).

29. Cerezo, M. et al. Translational control of tumor immune escape via the elF4F-STAT1-PD-L1 axis in melanoma. Nat. Med. 24, 1877-1886 (2018).

30. Feldman, M. E. et al. Active-site inhibitors of mTOR target rapamycin-resistant outputs of mTORC1 and mTORC2. PLoS Biol. 7, e38 (2009).

31. Gani, O. A. B. S. M. \& Engh, R. A. Protein kinase inhibition of clinically important staurosporine analogues. Nat. Prod. Rep. 27, 489-498 (2010).

32. Apsel, B. et al. Targeted polypharmacology: discovery of dual inhibitors of tyrosine and phosphoinositide kinases. Nat. Chem. Biol. 4, 691-699 (2008).

33. Kussie, P. H. et al. Structure of the MDM2 oncoprotein bound to the p53 tumor suppressor transactivation domain. Science 274, 948-953 (1996).

34. Sattler, M. et al. Structure of Bcl-xL-Bak peptide complex: recognition between regulators of apoptosis. Science 275, 983-986 (1997).

35. Moellering, R. E. et al. Direct inhibition of the NOTCH transcription factor complex. Nature 462, 182188 (2009).

36. Dazert, E. \& Hall, M. N. mTOR signaling in disease. Curr. Opin. Cell Biol. 23, 744-755 (2011). 
37. Wang, X. et al. Regulation of elongation factor 2 kinase by p90RSK1 and p70 S6 kinase. EMBO J. 20, 4370-4379 (2001).

38. Moerke, N. J. et al. Small-molecule inhibition of the interaction between the translation initiation factors elF4E and elF4G. Cell 128, 257-267 (2007).

\section{Figures}
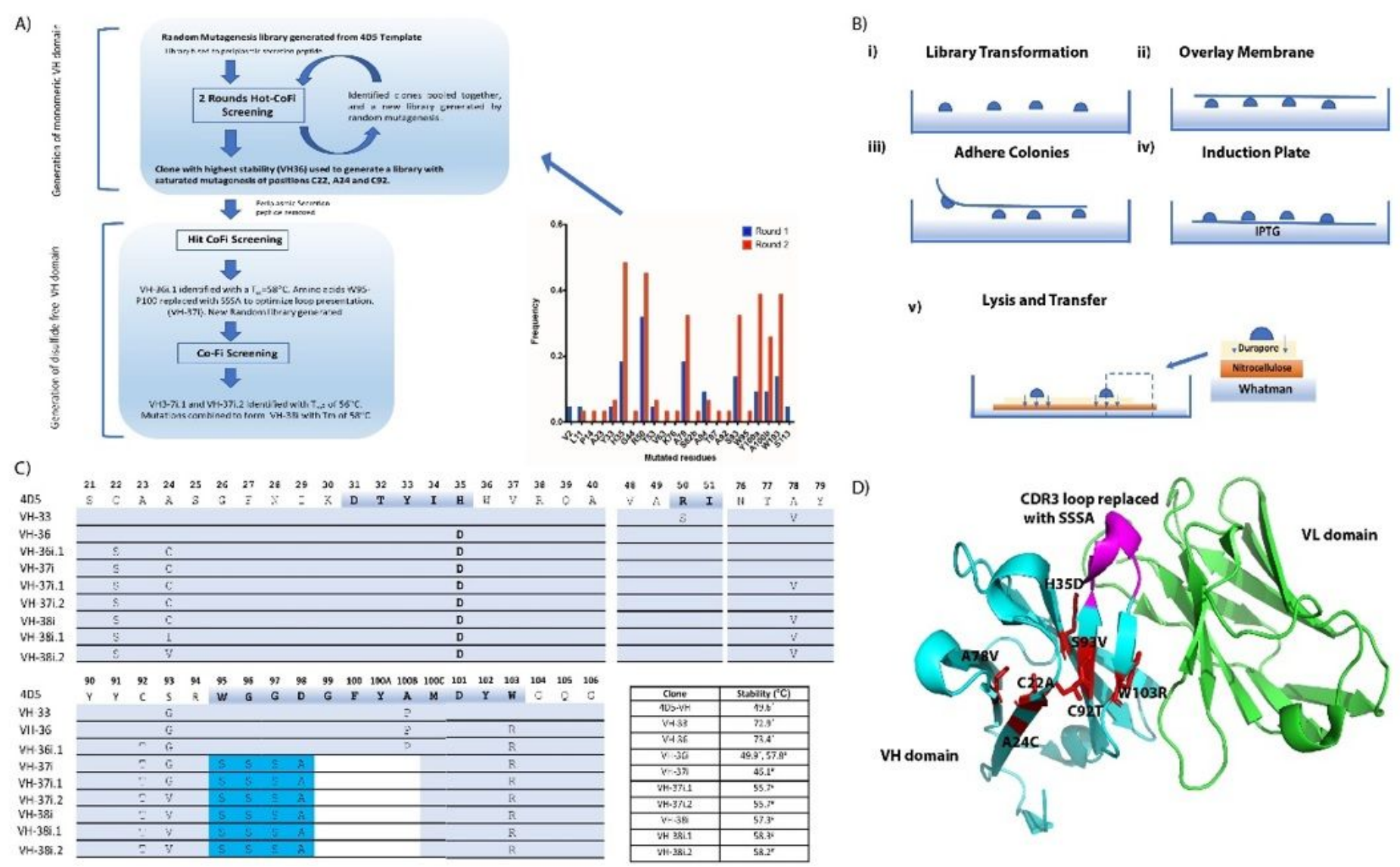

\section{Figure 1}

A) Flow-chart describing the directed evolution procedure, utilizing Hot-Cofi and Cofi selection techniques, to generate a disulphide-free autonomous antibody VH Domain suitable for phage display and intracellular eukaryotic expression applications. Inset: bar chart displays frequency of specific mutations isolated from the first 2 rounds of Hot-Cofi selection. B) Schematic illustration of the CoFi selection procedure. i) Cells transformed with the randomly mutagenized library are plated and grown on LB plates. ii) The filter membrane (Durapore) is overlaid on top of the colonies, iii) which adhere to the membrane and can be removed from the LD agar. iv) Protein expression is induced in the transformed bacterial colonies by placing the filter on IPTG containing LB plates. v) The cells are lysed, and soluble proteins diffuse through the filter membrane (Durapore) and are captured on the nitrocellulose membrane while inclusion bodies are retained on the filter membrane. The Whatman $3 \mathrm{MM}$ paper and the nitrocellulose membrane are both soaked in native lysis buffer to release the VH domain clones from the bacterial 
colonies. By probing the nitrocellulose membrane with antibodies, colonies expressing soluble proteins can be identified. If performing the Hot CoFi method, the induction plate is incubated at the desired temperature above the wild-type protein melting temperature at step iv), before transferring the Durapore membrane onto the top of the nitrocellulose membrane in step v). C) Summary of VH clones isolated in the directed evolution procedure outlined in $\mathrm{A}$ ) with their respective mutations and associated thermal stabilities. The $\mathrm{VH}$ domain clones are numbered using the Kabaat nomenclature system to denote residues and CDR positions (highlighted in blue in the 4D5 sequence). Only sections of the aligned VH domains containing mutations are shown. Residues highlighted in cyan were inserted to replace residues 95 - 100B in clones $\mathrm{VH}-37 \mathrm{i}$ and onwards. White space denotes residues deleted from the $\mathrm{VH}$ domain sequence as result of SSSA insertion. D) Structure of $\mathrm{VH}$ domain in complex with the $\mathrm{VL}$ domain, extracted from the FAB fragment crystal structure of Trastuzumab (PDB code: 6BAE), highlighting the location of the mutations found in $\mathrm{VH}-38 \mathrm{i}$ and $\mathrm{CDR} 3$ loop replacement. 
A)
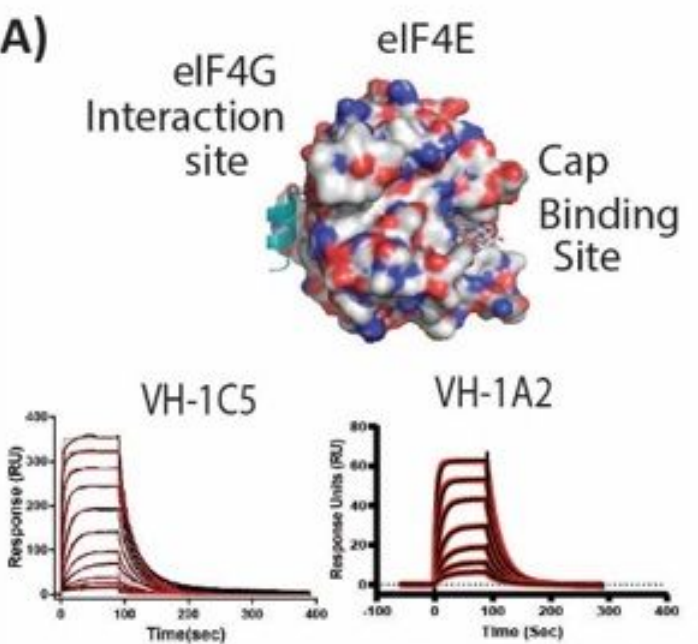

$K_{d}=154.2 \pm 70.3 n M$

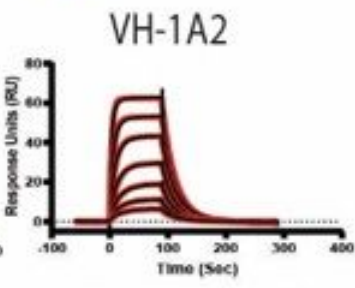

$\mathrm{K}_{\mathrm{d}}=115.2 \pm 4.4 \mathrm{nM}$
B)

eIF4E Interacting Sequences

eIFG1 LEEKKRYDREFLLGFQFIFA

4EBP1 GGTRIIYDRKFLMECRNSPV

$1 \mathrm{C5}$ VAKALNSSSPSFVVNTYSSFG

$1 A 2$ MAYSYPSSFSASSFDLYYA
C)

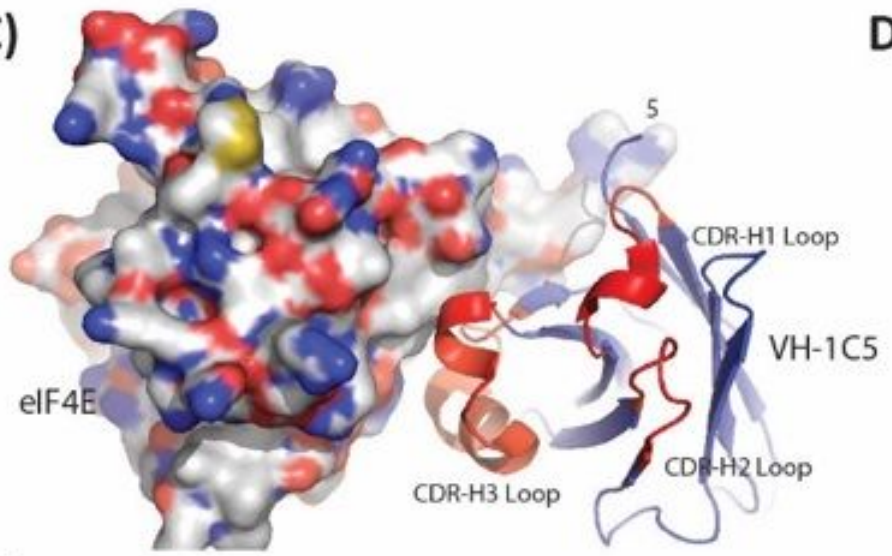

E)

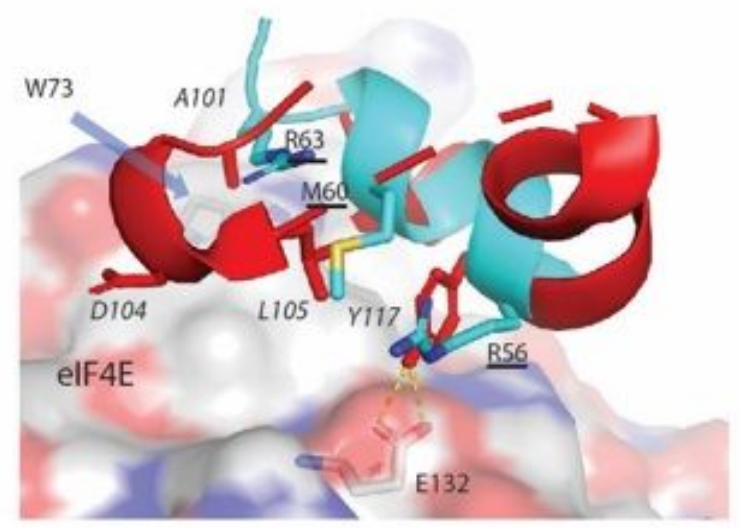

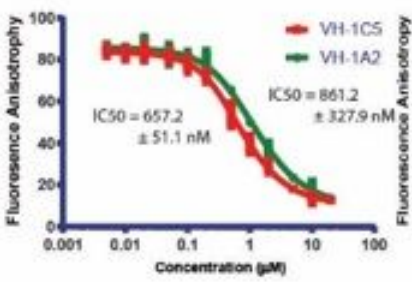
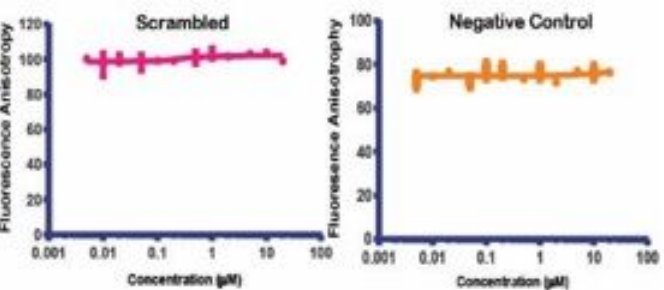

D)

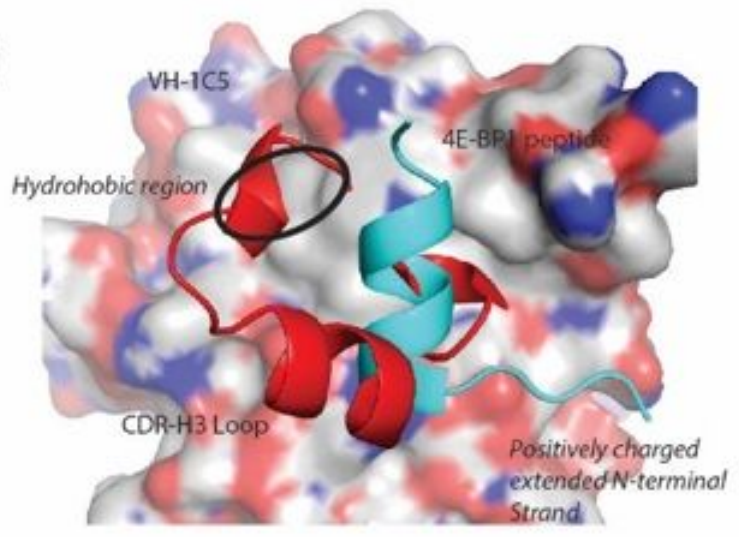

F)

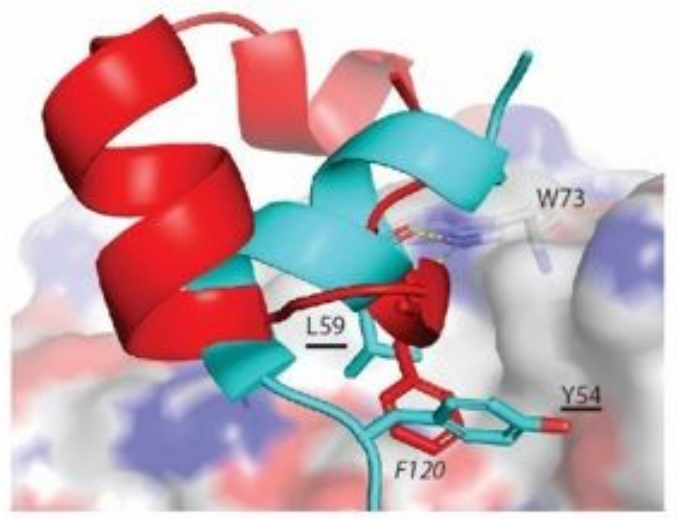

Figure 2

A) Surface and cartoon representation of elF4E showing the locations of the cap-binding and elF4G interaction sites. Surface plasmon resonance sensograms showing titrations of $\mathrm{VH}-1 \mathrm{C} 5$ and $\mathrm{VH}-1 \mathrm{~A} 2$ against elF4E immobilised via amine coupling on CM5 chips (see materials and methods). Binding and kinetic data are shown on the graphs. B) Comparison of the naturally occurring elF4E interacting sequences (4E-BP1 and elF4G1) with the selected VH domain library sequences (1A2 and 1C5), which were isolated from phage panning experiments against elF4E. Graphs show titrations of VH domains $1 \mathrm{C5}$ and $1 \mathrm{~A} 2$ against eiF4E in fluorescent polarization competition experiments using a FAM labelled elF4G1 
peptide (KKRYSRDFLLALQK-(FAM)). Derived dissociation constants are shown on the graph (see materials and methods). C) Crystal structure of elF4E in complex with VH-1C5. elF4E is shown using surface representation, whist $\mathrm{VH}-1 \mathrm{C} 5$ is shown using cartoon representation. The phage library selected CR3 loop insert that interacts with elF4E is highlighted in red, whilst the main fold of the VH domain is shown in cyan. Highlighted in oranges are close contacts that form between the VH domain and elF4E that lie outside the library insert region of the CDR3 loop. D) Overlay of the CDR3 library loop region (highlighted in red) with the elF4E interacting sequence from 4E-BP1 (highlighted in cyan). Both sequences adopt very different secondary structural folds to each other and have overlapping interaction sites with elF4E. The VH-1C5 CDR3 loop forms additional contacts with a hydrophobic region on the surface of elF4E that the 4E-BP1 peptide does not, whilst the positively charged N-terminal extended strand of 4E-BP1 Interacts with a negatively charged patch on elF4E not observed in VH1C5. E) Residues A101 and D104 of VH-1C5 form contacts with the hydrophobic region of elF4E, whilst L105 recapitulates the interactions of the M60 residue from 4E-BP1. Y117 from the CDR3 loop of VH-1C5 forms a hydrogen bond with E132, replacing the electrostatic interaction observed with R56 in 4E-BP1. F) The F120 residue found in VH-1C5 mimics the hydrophobic contacts made by L59 and Y54 of 4E-BP1 against elF4E.

Additionally, the conserved hydrogen bond formed between the carbonyl backbone of L59 with the indole side chain of W73 is repeated by the carbonyl backbone of F220. Both Y54 and L59 are critical components of the elF4E interaction motif with elF4E $(Y--L \varphi, \varphi$ is any hydrophobic residue). 

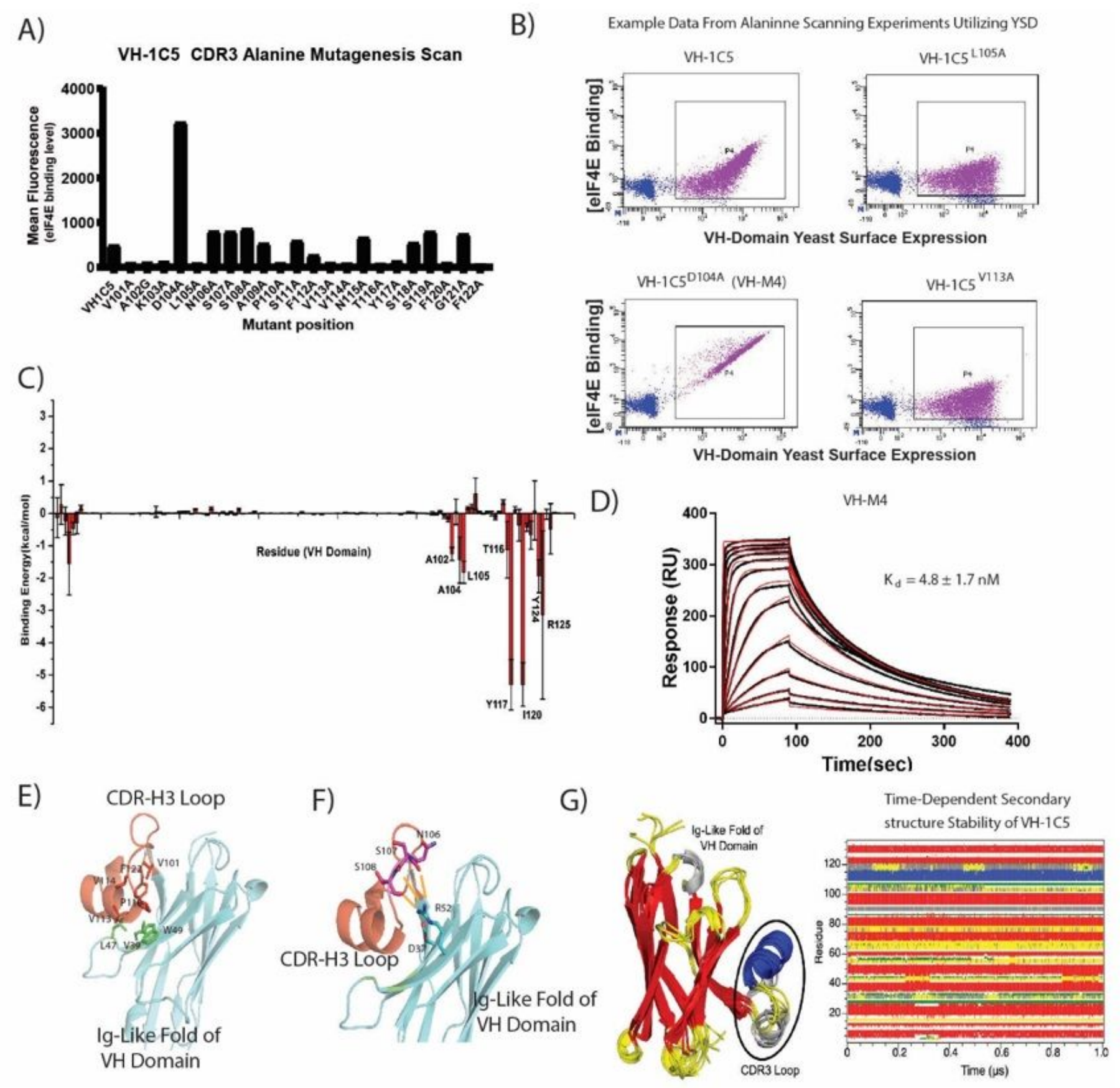

Figure 3

A) Scanning alanine mutagenesis was performed on the CDR3 loop of 1C5 using yeast display (YSD). Alanine mutations were made in individual yeast clones and tested for binding against fluorescently labelled elF4E (see materials and methods). Positions were alanine occurred were mutated to glycine instead. B) Example data from YSD alanine scanning experiments compared to $\mathrm{VH}-1 \mathrm{C} 5$. Mutations $\mathrm{VH}-$ 1C5 L105A and VH1C5 L105A V113A demonstrate experiments where binding to elF4E was lost, whilst VH-1C5 D104A shows an experiment where the alanine substitution resulted in increased binding to eIF4E. C) MD simulations were performed with elF4E complexed with $\mathrm{VH}-1 \mathrm{C} 5$, followed by residue-wise decomposition analysis of the intermolecular interaction. The residues which make $\geq-1.0 \mathrm{kcal} / \mathrm{mol}$ contribution to the binding energy are explicitly labelled in the plot (see materials and methods)..D) 
Surface plasmon resonance sensogram of VH-1C5E104D being titrated against elF4E immobilised via amine coupling on a CM5 chip (see materials and methods). Binding and kinetic data are shown on the graphs. E) The elF4E interacting CDR3 loop is highlighted in red. Residues shown in stick representation highlight critical amino acids that when mutated disrupt the hydrophobic cluster that stabilises the fold of the CDR3 loop (V101, V113, V114, P110 and F122), which is critical for binding elF4E. Residues highlighted in green depict amino acids that contribute to the hydrophobic cluster that are located in the main fold of the VH domain (L47, V39 and W49). F) The CDR3 loop is further stabilised by electrostatic interactions between R52 with the backbone carbonyls of N106, S107 and S108. Residue D32 orientates R152 into an optimal position to stabilise the CR3 loop by forming a salt-bridge with it. G)

Superimposition of representative structures from MD simulation of $\mathrm{VH}-1 \mathrm{C} 5$ domain unbound in solution. Graph depicts time dependent evolution of secondary structure of VH domain computed using DSSP algorithm. The CDR3 loop of $\mathrm{VH}-1 \mathrm{C} 5$ retains its bound conformation over the length of the simulation, indicating its inherent stability 
A)

B)

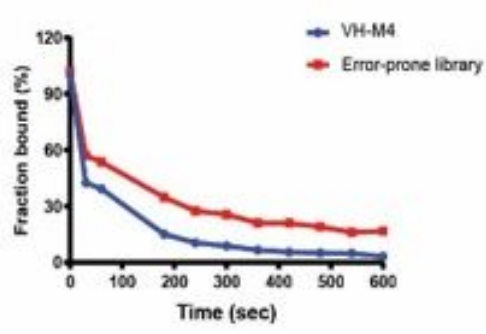

Kinetics Based Selection Strategy
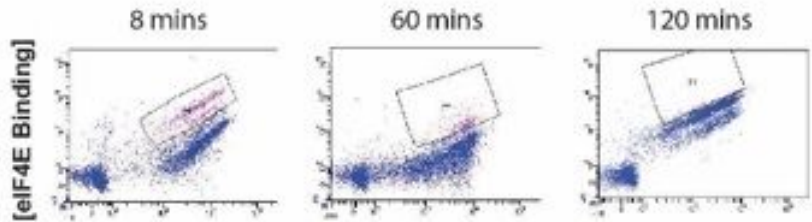

VH-Domain Yeast Surface Expression

C)

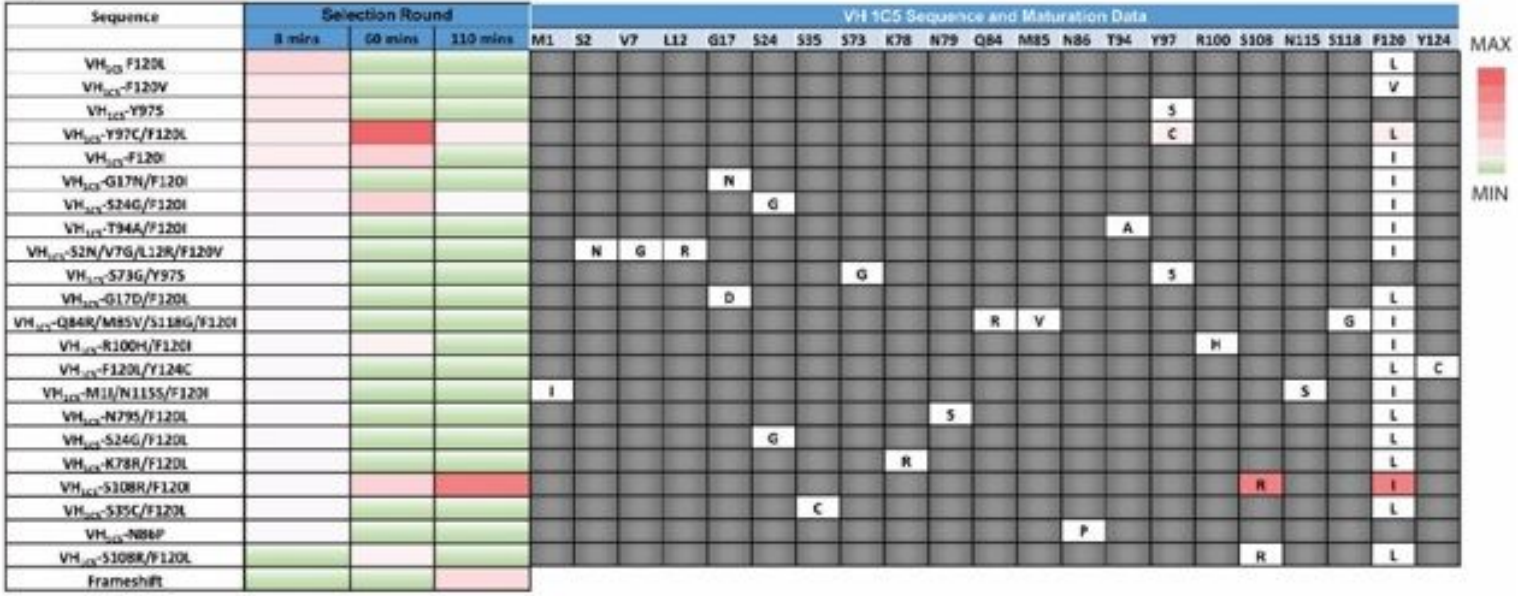

D)

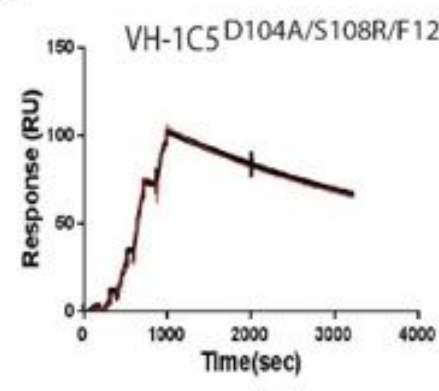

$K_{d}=0.057 \pm 0.004 n M$

E)

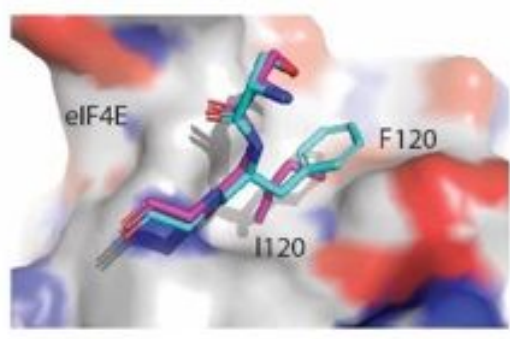

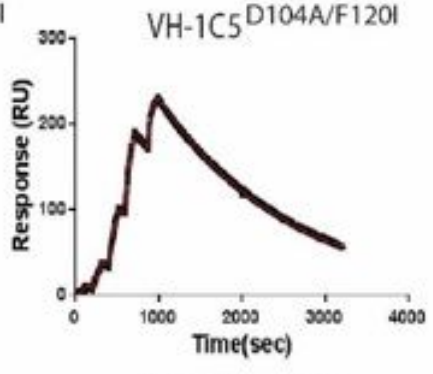

$K_{d}=0.64 \pm 0.06 \mathrm{nM}$

F)

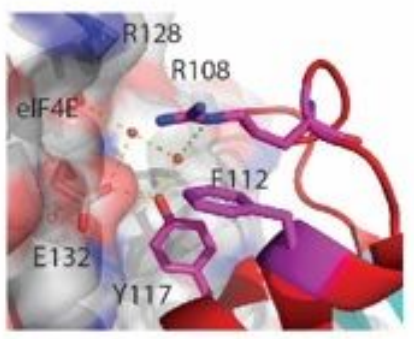

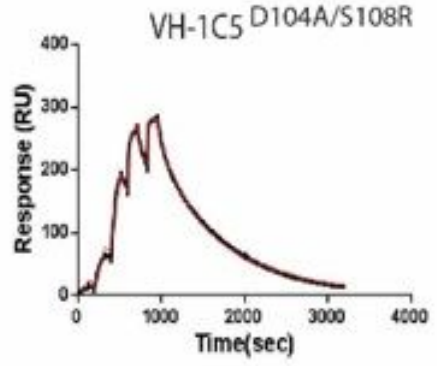

$K_{8}=0.94 \pm 0.04 \mathrm{nM}$

G)

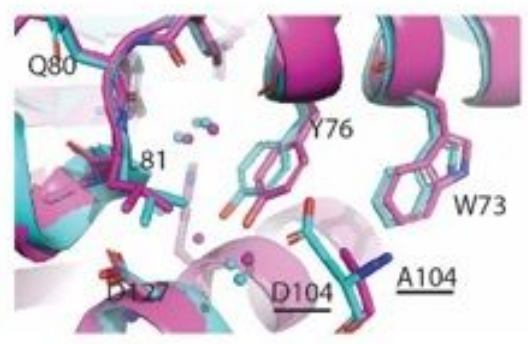

Figure 4

A) Dissociation rate of $\mathrm{VH}-1 \mathrm{C} 5 \mathrm{D} 104 \mathrm{~A}$ was compared to the error prone library, which was generated using VH-1C5D104A as template, to determine optimal competition time for dissociation rate engineering. Optimal competition time was approx. 8 mins. B) High affinity VH-1C5D104A variants were selected via three rounds of kinetic selection. Initial selectants were isolated using flow cytometry after competition with excess non-fluorescently labelled ligand after 8 mins. Selectants were then amplified and then 
kinetically selected after 60 mins. This was then repeated a third time with an incubation period of 110 mins. C) The table shows the analysis of the sequencing data from each round of selection with associated mutations. Approximately 30-50 yeast isolates were sequenced each round. The left-hand side of the table shows the enrichment of specific sequences with each round. Green indicate sequences that do not occur in that round of sequencing. The right-hand side of the table shows the specific mutational data for each yeast clone sequences. The most enriched sequence from the last round is highlighted in red. D) Surface plasmon resonance sensograms of VH1C5D104A/S108R/F120I, VH-1C5D104A/F120I and VH-1C5D104A/S108R being titrated against elF4E immobilised via amine coupling on CM5 chips (see materials and methods). Binding and kinetic data are shown on the graphs. E) Mutation of F120I that occurs in the high affinity VH-1C5D104A/S108R/F120I domain results in improved surface recognition of elF4E. F) The arginine that replaces S108 results in several new interactions. It engages a structured water network that forms hydrogen bonds with the backbone carbonyl of R128 and with both E132 and Y117. Additionally, it stabilises the fold of the CDR3 loop further by forming a cation- $\pi$ interaction with F112. G) Overlay of VH-1C5:elF4E (cyan) and VH-1C5D104A/S108R/F120l:elF4E (magenta) complex crystal structures showing the conformation change induced in $\mathrm{Y} 76$ of elF4E upon mutation of D104A. Associated structured water network also migrates with change in Y76 conformation. 
A

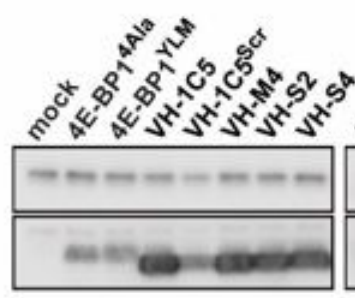

WCL
B

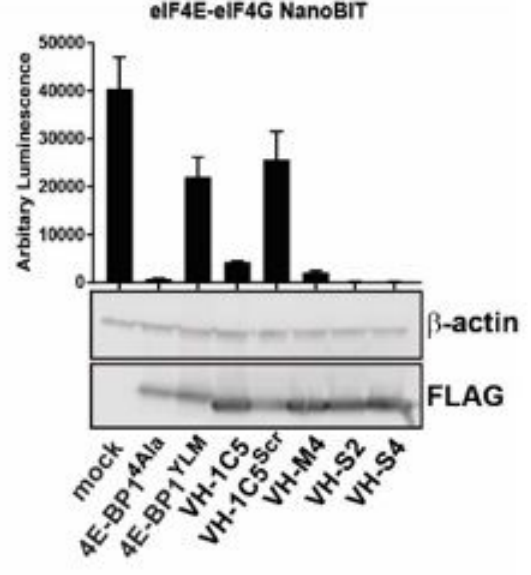

C
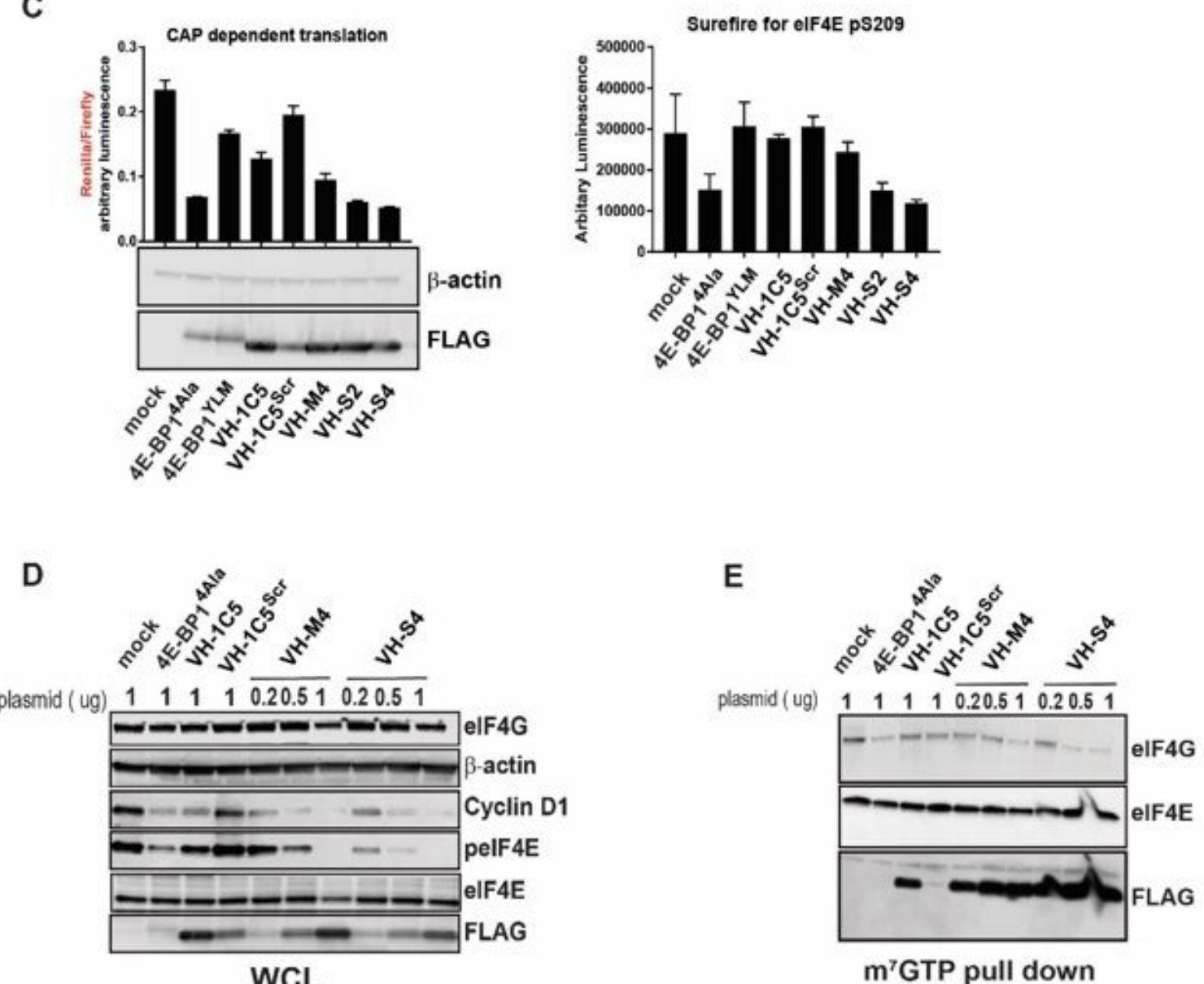

Figure 5

A) Western blot analysis of 293FT cells transfected fo 48 hrs with FLAG tagged empty vector (Mock), 4EBP1 and anti-EIF4e VH mutants, in whole cell lysate (Left, WCL) and FLAG immunoprecipitation analysis (right). Level of endogenous elF4E was detected with anti-elF4E. B) SmBiT-elF4E and elF4G604646-LgBiT constructs were co-transfected into HEK293 cells with either empty vector (Mock) or vectors containing 4EBP1 or VH mutants (as indicated) for 48 hrs. After 4 hours of incubation, the luciferase 
activities of the reconstituted NanoBit protein were measured in living cells as described in Material and Methods. All values represent mean +/- SD ( $n=3)$. C) A bicistronic luciferase reporter (left panel), which measures the relative amount of cap-dependent translation (Renilla) to cap-independent translation (Firefly), was co-transfected with indicated plasmid. Renilla and Firefly luciferase activity was measured 48 hours post transfection and plotted as a ratio-metric value. The Renilla luciferase is under the control of a GC rich 5UTR whose activity correlated to cap dependent translation, whilst the Firefly luciferease is regulated by an internal ribosomal site (IRES). Lysates were prepared from the transfected cells and blotted for the presence of the respective constructs (below left-hand graph). In the right-hand graph, the lysates from the bicistronic assay were also probed for serine 209 phoshorylation on eiF4E with Alpha screen Surefire assay. All values represent mean $+/-S D(n=3)$. D) Hela cells were transfected with indicated DNA at different plasmid concentration for $48 \mathrm{hrs}$ and level of cyclin D1, Phospho elF4E, elF4E, eIF4G and FLAG tagged proteins were analysed with the indicated antibodies by western blot. E) Lysates from transfected HEK293 cells were used to perform m7GTP pull down followed by western blot analysis. In whole cell lysate (Left, WCL) and m7GTP pull down analysis (right) of elF4G, elF4E and FLAG. Level of 4EBP and VH mutants were detected in western blot analysis for each experiment with anti-FLAG antibodies and loading was assessed with anti-actin antibody 
A
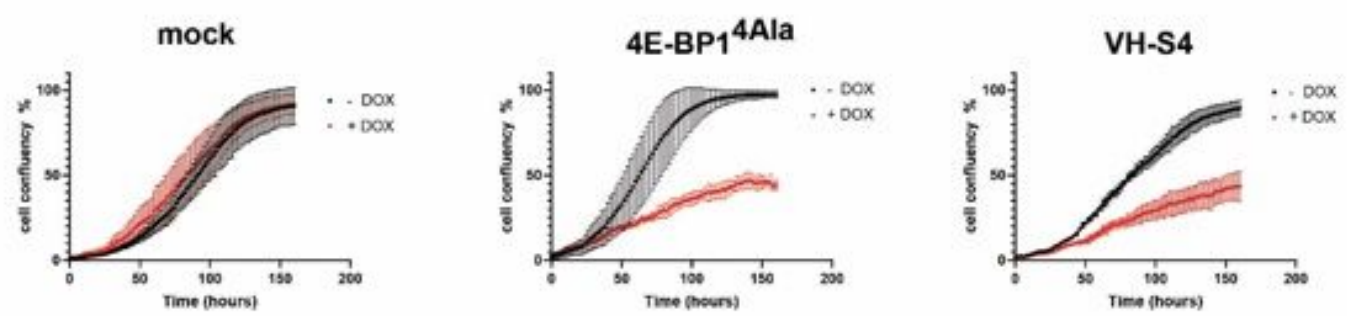

B
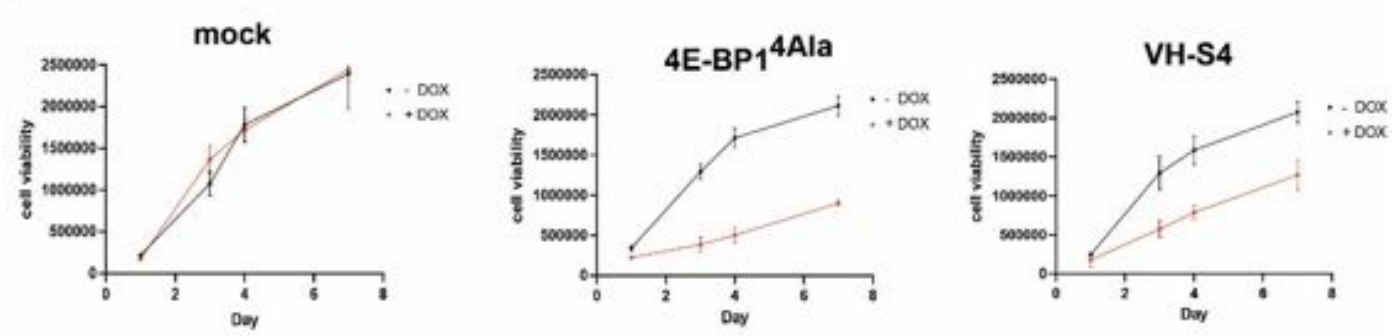

C

D
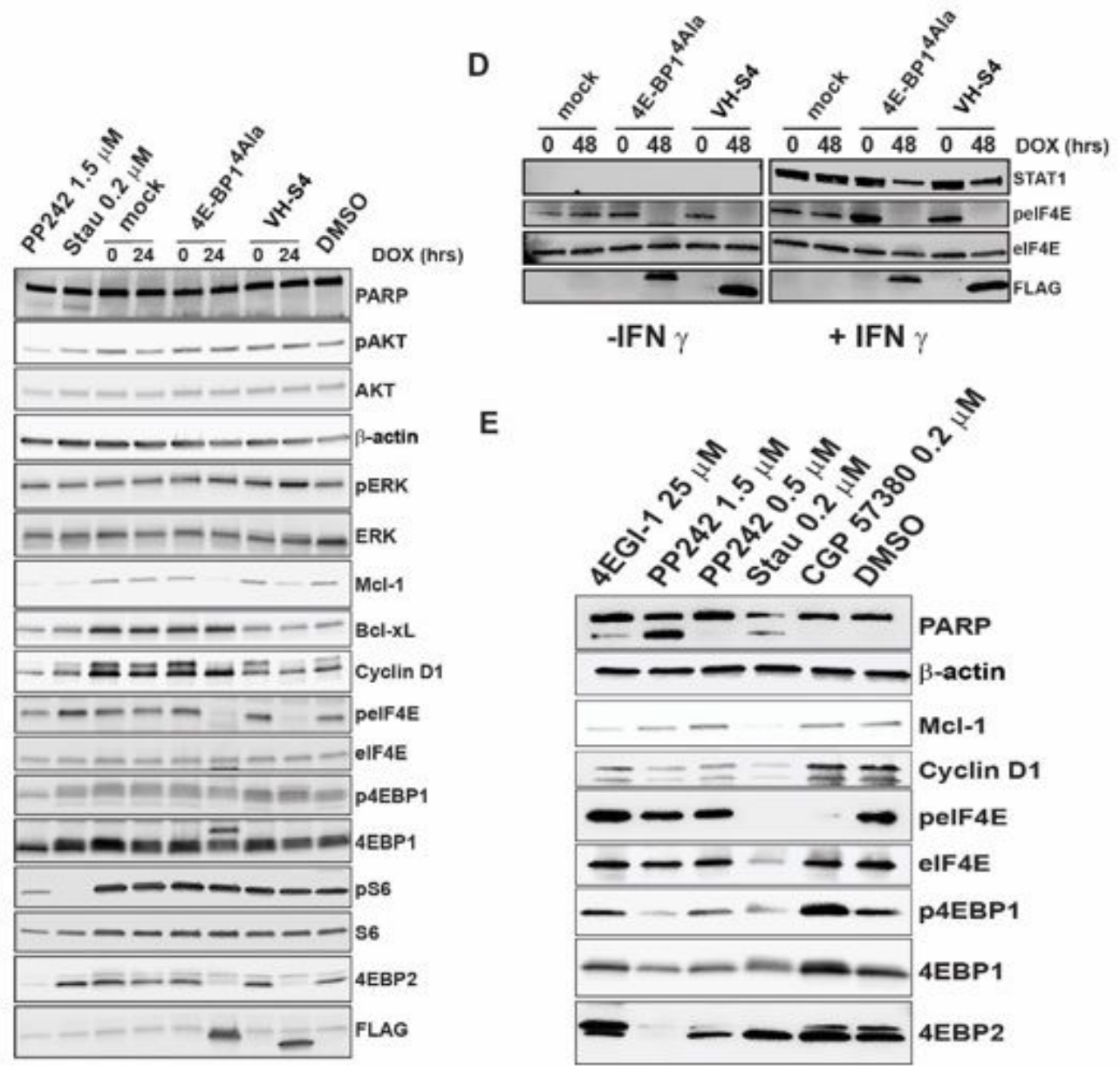

E

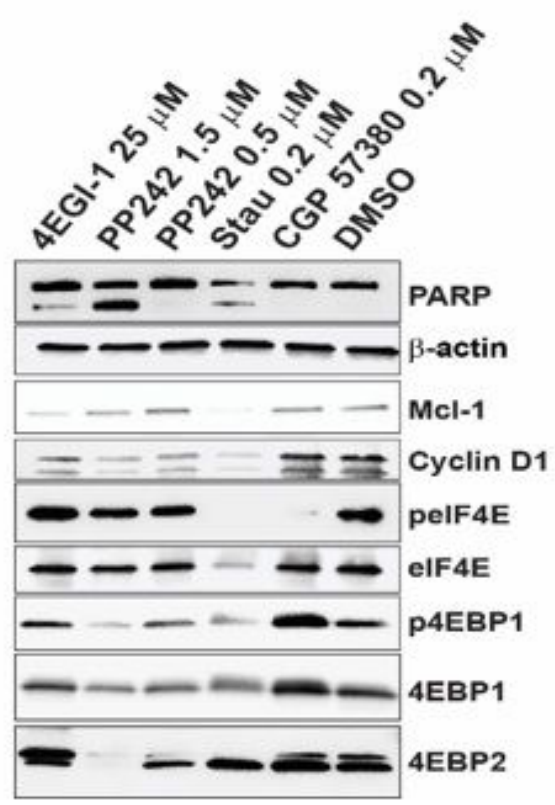

\section{Figure 6}

A) A375 Mock, 4E-BP14Ala and VH-S4 inducible cell line confluence was measured in presence or absence of doxycycline for the indicated time (hrs) using an Incucyte (EssenBiosciences). B) A375 stable cell lines treated as in A) were assayed for viability over the indicated time (days). All values represent mean +/- SD ( $n=3)$. C) Inducible A375 cells harbouring Mock, 4E-BP14Ala and VH-S4 were incubated for 24 hrs with or without $1 \mathrm{ng} / \mathrm{ml}$ of Doxycycline. Lysates were analysed by western blot using the anti- 
bodies indicated in the blot (for further details see materials and methods). B-Actin was used as a loading control. (D) A375 inducible stable cell lines were simultaneously incubated with or without doxycycline and where either treated with or without IFN- $\gamma(50 \mathrm{ng} / \mathrm{ml})$ for $48 \mathrm{hrs}$. After cell lysis, extracts were analysed by western blot and protein levels for STAT1, Phosho-elF4E, eiF4E and FLAG tagged 4E-BP14Ala and $\mathrm{VH}-\mathrm{S} 4$ were measured. E) A375 cells were treated with the indicated compounds at the labelled concentrations for 72 hours with a residual DMSO concentration of $1 \%(\mathrm{v} / \mathrm{v})$. Lysates were analysed by western blot using the anti-bodies indicated in the blot (for further details see materials and methods).

\section{Supplementary Files}

This is a list of supplementary files associated with this preprint. Click to download.

- SupplementaryDataV5.docx 